\title{
Caracterización de pacientes con enfermedad de Parkinson en un centro de referencia de la ciudad de Bogotá, Colombia
}

\section{Characterization of patients with Parkinson's disease in a reference center of Bogotá, Colombia}

Lina M. Ariza-Serrano (1), Jairo Guerrero-Vega (1), Paola Ortíz (2), Claudia L. Moreno-Lopez (3)

\section{RESUMEN}

INTRODUCCIÓN: la enfermedad de Parkinson es una de las enfermedades neurodegenerativas más prevalentes a nivel mundial. Se han documentado las características clínicas de presentación de la enfermedad, además de los síntomas motores y no motores. En nuestra población no hay datos al respecto.

OвJETIvo: describir las características demográficas y clínicas de una cohorte de pacientes con enfermedad de Parkinson, evaluados en un centro de referencia de movimientos anormales de la ciudad de Bogotá, Colombia.

Materiales y MÉtodos: estudio descriptivo retrospectivo de los pacientes con enfermedad de Parkinson que asisten a la consulta de movimientos anormales. Se observaron todos los pacientes registrados en la base de datos de enero de 2013 hasta junio de 2015 y se revisaron las variables sociodemográficas, los síntomas motores y no motores y se analizaron de acuerdo al tiempo de evolución de la enfermedad. Todos los datos fueron analizados en el programa SPSS, en donde se establecieron frecuencias, promedios y proporciones.

Resultados: se analizaron en total 446 pacientes, de los cuales el $50.7 \%$ son mujeres. La edad promedio encontrada fue de 68 años. Encontramos en ellos, síntomas pre-motores como hiposmia en un $33.6 \%$ y alteraciones del sueño REM en un $32 \%$. Los síntomas motores experimentados como de presentación inicial fueron: temblor $(62.3 \%)$, bradicinesia $(15.9 \%)$, rigidez (12.6 \%), dificultad para caminar $(8.1 \%)$, alteración del equilibrio $(1.6 \%)$ y caídas $(1.6 \%)$.

Conclusión: en la población estudiada se encontraron frecuencias de patrones motores similares con los registros internacionales, sin embargo, las características demográficas y las manifestaciones no motoras tuvieron algunas diferencias.

PALABRAS CLAVE: enfermedad de Parkinson, frecuencia, síntomas motores, síntomas no motores, Colombia (DeCS).

\section{SUMMARY}

InTRODUCTION: Parkinson's disease is one of the most prevalent neurodegenerative diseases worldwide. It's clinical presentation, motor and non-motor signs have been described previously. In our population, we don't know the frequency of those signs and symptoms.

OвJестіve: Describe the clinical presentation, motor and non-motor signs of a cohort of patients with Parkinson's disease, that were evaluated in a movement disorders center of reference, at Bogotá, Colombia.

Materials and methods: Descriptive retrospective study of patients with Parkinson's disease, who went to the movement disorders clinic between January 2013 and June 2015. Patients were organized depending on the time from the onset of the disease. Socio-demographic variables, motor and non-motor signs and symptoms where studied. Frequencies, mean and proportions were analyzed using SPSS 22 version.

REsults: 446 patients were analyzed, from which $50.7 \%$ were women. The mean age found was 68 years old. We found hiposmia in 33.6\% of patients and abnormalities in REM sleep in 32\% of them. The first motor symptoms noticed by the patient were tremor $(62.3 \%)$, bradykinesia $(15.9 \%)$, stiffness $(12.6 \%)$, difficulty walking $(8.1 \%)$, balance problems (1.6\%) and falls (1.6\%).

(1) Residente de Neurología, Universidad del Rosario, Fundación Cardioinfantil, Bogotá, Colombia

(2) Neuróloga, Fundación Cardioinfantil, Bogotá, Colombia

(3) Neuróloga, especialista en movimientos anormales, Fundación Cardioinfantil, Bogotá, Colombia

Recibido: 6/06/16. Aceptado: 5/09/16.

Correspondencia: Lina M. Ariza-Serrano, ariza.linamaria@gmail.com 
ConCLusion: Parkinson's disease is the second most frequent neurodegenerative disease worldwide. Its clinical presentation is diverse, and in our population we have never study the patterns of presentation. We found similar frequencies compared with the international registries of this disease, with some difference in demographic and non-motor symptoms.

KEY WORDS: Parkinson's disease, frequency, motor symptoms, non-motor symptoms, Colombia (MeSH).

\section{INTRODUCCIÓN}

La enfermedad de Parkinson es la segunda enfermedad neurodegenerativa más frecuente a nivel mundial, después de la enfermedad de Alzheimer ${ }^{1,2}$. La prevalencia a nivel mundial se calcula que es de 150 por cada 100.000 habitantes, y estas cifras aumentan si hay ajustes de edad, por cada década de más que tenga el paciente ${ }^{3}$. En los países europeos, la prevalencia varía desde 65.6 por cada 100.000 hasta 12.500 por cada 100.000 , y la incidencia varia desde 5 por cada 100.000 , hasta 346 por cada 100.0002 . Se cree que estas frecuencias también varían dependiendo de la raza, edad y género, siendo más frecuente en hispanos, hombres y mayores de 60 años 4 .

En Colombia, se han realizado dos estudios que muestran la prevalencia de esta enfermedad5,6. En el primero (estudio EPINEURO), realizado en el año 2003, se mostró una prevalencia de 4.4 por cada 1.000 habitantes5, y en el año inmediatamente posterior, en el departamento de Antioquia se encontró una prevalencia de 30.7 por cada 100.000 habitantes $^{6}$.

La enfermedad de Parkinson, se considera como una causa multifactorial, genéticas en un $10 \%$ de los casos y medioambientales hasta en un $90 \%{ }^{7,3}$, ocasionando cambios imagenológicos localizados en la sustancia nigra mesencefálica de las personas afectadas ${ }^{8}$. Para el abordaje diagnóstico de esta enfermedad, según los criterios del Banco de cerebros de Londres, estos pacientes deben cursar con bradicinesia, y al menos uno de los siguientes tres síntomas motores: rigidez muscular, temblor en reposo o inestabilidad postural9. Síntomas autonómicos, cognitivos, del sueño REM, psiquiátricos y alteraciones sensitivas también han sido descritas, y son de gran importancia diagnóstica por el impacto en la calidad de vida de estos pacientes ${ }^{10}$. Se cree que el estado cognitivo y mental tienen un mayor impacto sobre la calidad de vida, que los síntomas motores ${ }^{11}$.

La enfermedad de Parkinson tiene una expresión clínica variable, y se han descrito dos grandes subtipos: uno caracterizado por el temblor como su síntoma predominante y otro donde predominan los síntomas rígido-acinéticos, pudiendo incluso carecer completamente de temblor este último. Algunos autores han descrito un menor compromiso cognitivo, un inicio más temprano de la enfermedad y una progresión más lenta en aquellos pacientes con subtipos donde predomina el temblor ${ }^{12}$. El estudio ReMePARK realizado en la población mexicana mostró como síntoma predominante el temblor en el $65.5 \%$ de los sujetos, seguido de rigidez-bradicinesia en el $30 \% 13$. En Bogotá no se cuenta con datos epidemiológicos que puedan corroborar estos hallazgos para ser aplicados en nuestra población.

Por otra parte, los síntomas no motores también son importantes en la enfermedad de Parkinson, aunque no se encuentren en los criterios diagnósticos, pueden servir de herramienta para apoyar el diagnóstico de la enfermedad. Es por esto que se han realizado estudios como el PRIAMO para evaluar su impacto y frecuencia en la enfermedad de Parkinson. En este estudio se encontró que hasta un $98.6 \%$ de los pacientes con EP presentaron al menos 1 síntoma no motor, siendo la fatiga el síntoma más frecuente ${ }^{14}$.

En este estudio, descriptivo, retrospectivo, se establecieron frecuencias y promedios y tiene una importancia epidemiológica y clínica, que aportará datos útiles en la práctica clínica acerca del comportamiento normal de nuestra población.

\section{MATERIALES Y MÉTODOS}

Estudio descriptivo, retrospectivo, tomando la información de la base de datos de los pacientes con enfermedad de Parkinson que asisten a consulta a un centro de referencia de movimientos anormales de la ciudad de Bogotá, Colombia, desde enero de 2013 hasta junio de 2015.

Todos los pacientes seleccionados de la base de datos, cumplían el criterio diagnóstico del Banco de cerebros de Londres, es decir, los pacientes debían cursar con bradicinesia y al menos uno de los siguientes tres síntomas motores: rigidez muscular, temblor en reposo o inestabilidad postural, y en cada uno de ellos fueron revisados los criterios que exclusión que recomendados.

De esta base de datos se tomaron datos de variables sociodemográficas y clínicas. De las variables clínicas la hiposmia, trastornos del sueño REM, síntomas sensitivos, cognitivos, descontrol de impulsos y depresión fueron determinados por presencia o ausencia en el momento de la valoración por clínica inicial de movimientos anormales. En el caso de la hiposmia, no se utilizó ninguna escala o método que objetivara dicho hallazgo. Temblor, bradicinesia, rigidez 
e inestabilidad postural, eran síntomas que se preguntaron si habían estado al inicio de la enfermedad.

Se descartaron aquellos pacientes que no tenían toda la información completa o quienes tenían duda diagnóstica (parkinsonismos).

Para el análisis estadístico, en las variables de tipo cualitativo se utilizó distribuciones de frecuencia y distribuciones porcentuales, en las variables de tipo cuantitativo medidas de tendencia central como el promedio y la mediana con su respectiva medida de dispersión. El procesamiento de la información se realizó usando los paquetes estadísticos SPSS versión 22.

Este estudio no requirió la firma de consentimiento informado, dado que es un estudio retrospectivo, sin intervención.

\section{RESULTADOS}

Un total de 456 pacientes se encontraron en la base de datos, 10 pacientes fueron excluidos (5 no tenían los datos completos y 5 tenían duda diagnóstica al momento de la revisión de los datos). Finalmente se analizaron los datos de 446 pacientes. El $50.7 \%$ de los pacientes eran de sexo femenino; la mediana de la edad fue 70 (RIC 30 - 97). El tiempo medio de evolución de la enfermedad al momento de la primera valoración, fue de 60 meses (DE \pm 71.1$)$. Tabla 1.

Síntomas pre-motores de la enfermedad: dentro de los síntomas pre-motores hallados se documentó hiposmia y trastornos del sueño REM en un 33.6 y $32 \%$ respectivamente, tabla 2. Cuando se analizó la presencia de estos síntomas según la edad, se observó que los pacientes jóvenes

Tabla 1. Características de la población.

\begin{tabular}{ll}
\hline Edad & $\mathbf{N}(\%)$ \\
$30-50$ años & $35(7.8)$ \\
$51-65$ años & $133(29.8)$ \\
$66-75$ años & $144(32.3)$ \\
$>75$ años & $134(30)$ \\
\hline Sexo (mujeres) & $226(50.7)$ \\
\hline
\end{tabular}

Años con la enfermedad en el momento de la primera valoración

$\begin{array}{ll}<1 \text { año } & 57(12.8) \\ 1-5 \text { años } & 174(39) \\ 5-10 \text { años } & 133(29.8) \\ >10 \text { años } & 82(18.4)\end{array}$

son los que menos la manifiestan, a diferencia de aquellos de 66 a 75 años en los que un $36.3 \%$ refirieron hiposmia, y un $35.9 \%$ trastornos del sueño REM. Tabla 2. Síntomas pre-motores en pacientes con enfermedad de Parkinson.

Síntomas motores de la enfermedad: los síntomas motores referidos por el paciente como síntoma inicial de la enfermedad fueron: temblor $(62.3 \%)$, bradicinesia $(15.9 \%)$, rigidez $(12.6 \%)$, dificultad para caminar $(8.1 \%)$, alteración del equilibrio $(1.6 \%)$ y caídas $(1.6 \%)$. Figura 1.

El temblor fue descrito como síntoma inicial en el 12.6 $\%, 40.3 \%, 32.4 \%$ y $14.7 \%$ del total de pacientes que referían este síntoma, según el tiempo de evolución de la enfermedad menor a 1 año, de 1 a 5 años, de 5 a 10 años o mayor de 10 años respectivamente. La bradicinesia fue descrita como síntoma cardinal, de presentación inicial y de mayor importancia en un $14.1 \%$ de los pacientes con menos de 1 año de evolución, en el $35.5 \%$ de aquellos personas en los primeros 5 años, $26.8 \%$ de 5 a 10 años y $23.9 \%$ de aquellos con más de 10 años de evolución de la enfermedad. La rigidez fue de aparición temprana en un total de 56 pacientes, de los cuales el 7.1\% tenían menos de 1 año de evolución de la enfermedad, el $33 \%$ se encontraba en los primeros 5 años de evolución, el 39.3 \% tenían de 5 a 10 años y el $19.6 \%$ llevaban más de 10 años de curso de la misma.

Con respecto a los grupos etarios, el temblor también fue el síntoma referido con mayor frecuencia. En $4.9 \%$ de los pacientes jóvenes de 30-50 años, $13.7 \%$ de aquellos de 51 a 65 años, $21.7 \%$ de 66 a 75 años, y $22 \%$ de los pacientes mayores de 76 años, el temblor fue descrito como síntoma inicial. La bradicinesia tuvo una presentación temprana en el

Tabla 2. Síntomas pre-motores.

\begin{tabular}{ll}
\hline Síntomas pre-motores & N (\%) \\
\hline Hiposmia & $146(33.6)$ \\
Según la edad del paciente & \\
30-50 años & $8(5.5)$ \\
$51-65$ años & $45(30.8)$ \\
$66-75$ años & $53(36.3)$ \\
$>76$ años & $40(27.4)$ \\
\hline Trastornos del sueño REM & $142(32)$ \\
según la edad del paciente & \\
30-50 años & $7(4.9)$ \\
$51-65$ años & $42(29.6)$ \\
$66-75$ años & $51(35.9)$ \\
>76 años & $42(29.6)$ \\
\hline
\end{tabular}


$1.1 \%$ de los pacientes de 30-50 años, y llegó a ser hasta del $7.4 \%$ en aquellos de 51 a 65 años. El $1.1 \%$ de los pacientes de 30 a 50 años, $3.8 \%$ de 51 a 65 años y $5.6 \%$ de aquellos de 66 a 75 años refirieron rigidez como síntoma inicial.

Otros síntomas de la enfermedad: durante el curso de la enfermedad, 81 pacientes describieron algún tipo de alteración sensitiva. Aquellos pacientes con más de 5 años de evolución de la enfermedad fueron los que más presentaron esta sintomatología en un $38.3 \%$.

Los cambios cognitivos también fueron evaluados a través del MMSE y confirmados con valoración neuropsicológica. Fueron pocos los casos que cursaron con esta entidad. Se documentaron cambios demenciales en 3 pacientes de más de 10 años de evolución de la enfermedad, en otros 3 pacientes de más de 5 años de evolución de la enfermedad, y en 2 pacientes que se encontraban en los primeros 5 años de evolución de la enfermedad. Ningún paciente de menos de 1 año de evolución de la enfermedad de Parkinson cursó con trastorno del control de impulsos. De un total de 5 pacientes que cursaron con este trastorno, 2 pacientes se encontraban en los primeros 5 años de la enfermedad, otros 2 pacientes se encontraban en los primeros 10 años, y 1 pacientes tenía mas de 10 años de evolución de esta entidad. Las alteraciones en el estado de ánimo también fueron evaluadas. Veintiún pacientes en los primeros 5 años, 22 pacientes en los primeros 10 años, y 9 pacientes de más de 10 años cursaron con síntomas de trastorno depresivo mayor. Figura 2.

\section{DISCUSIÓN}

La enfermedad de Parkinson, como ya se ha mencionado, tiene una presentación clínica heterogénea ${ }^{15}$, que se ha visto reflejada en nuestra población.

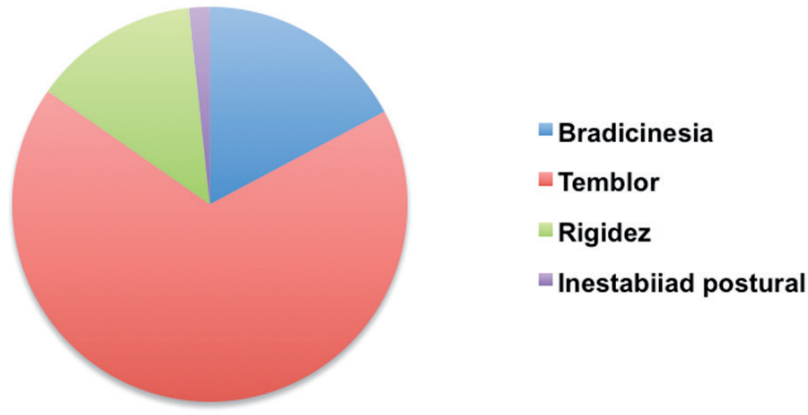

Figura 2. Síntomas motores de presentación inicial en pacientes con Enfermedad de Parkinson.

Los resultados encontrados tienen algunas características que difieren con lo reportado en las diferentes series nacionales e internacionales $13,16,17,18$, encontrando las principales diferencias en la variable de género, dado que hay mayor prevalencia en la población femenina, hallazgos que no se correlacionan con los estudios previamente realizados, donde se documenta una mayor prevalencia en hombres $13,16,17,18$.

La edad media de presentación (68.7 años) es equiparable a la reportada en la población española de la cohorte ELEP (66.2 años) ${ }^{16}$, e incluso en la población del estudio caleño (72.3 años) ${ }^{17}$. El tiempo promedio de evolución de la enfermedad hasta la primera valoración por la clínica de movimientos anormales fue de 82 meses.

El síntoma motor predominante fue el temblor, presente en el $62,3 \%$ de los sujetos, seguido de fenómenos rígido-acinéticos en $28,5 \%$, y menos frecuente los trastornos de la marcha en $8.1 \%$, hallazgos comparables con lo

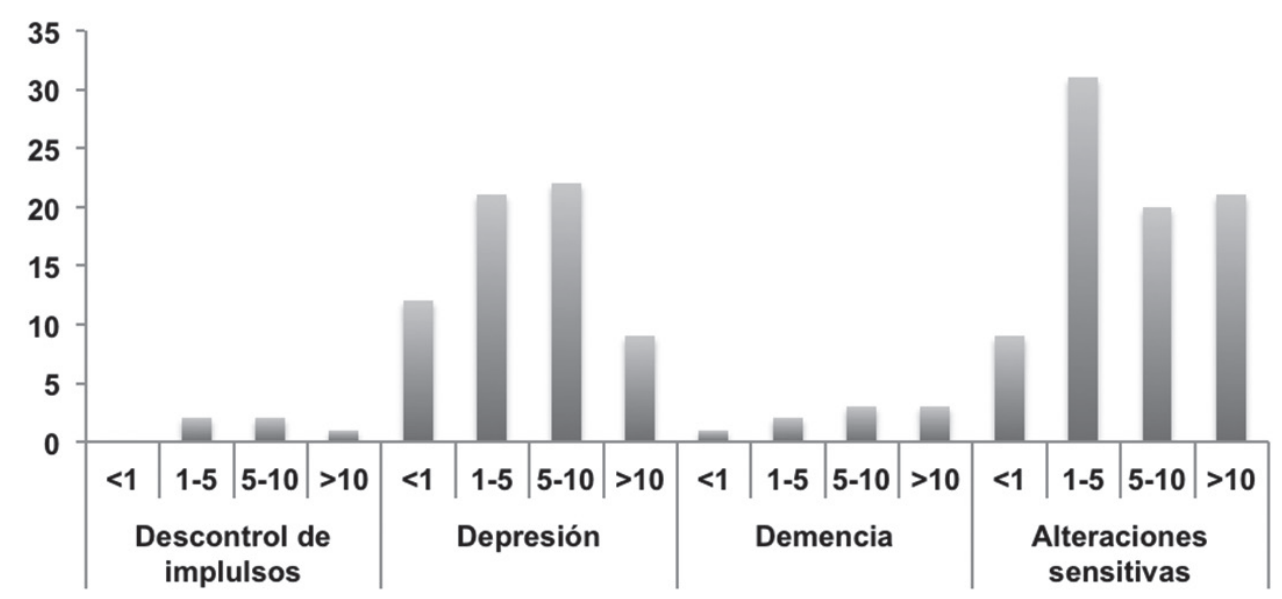

Figura 1. Número de casos que cursaron con síntomas No-Motores, según los años de evolución de la enfermedad. 
encontrado en la población mexicana ${ }^{19}$. Comparando con el estudio caleño, se conserva el orden de frecuencia de los síntomas motores al momento del diagnóstico, sin embargo el porcentaje de pacientes con temblor es menor (51.2\%), y el porcentaje de pacientes con inestabilidad postural fue notablemente mayor $(19.5 \%)^{17}$.

Independientemente de la edad de presentación y tiempo de evolución de la enfermedad, el subtipo clínico que predominó fue el de temblor. En los sujetos con menos de 1 año de evolución de la enfermedad y en menores de 50 años se observó un menor compromiso axial y de fenómenos rígido-acinéticos.

Con lo que refiere a los síntomas no motores, el más frecuente reportado fue la hiposmia, presente en $33.6 \%$ de los pacientes, seguido de los trastornos de conducta del sueño REM en $32 \%$, siendo estos hallazgos discretamente mayores a los reportados en el estudio PRIAMO, con $26.9 \%$ y $29.6 \%$ respectivamente ${ }^{14}$. Se destaca que estos síntomas no motores tuvieron su mayor frecuencia en pacientes de mayor edad, principalmente en el grupo de pacientes mayores de 65 años, y que estos síntomas no motores tuvieron una menor frecuencia en los sujetos con menos de 1 año de evolución de la enfermedad, hallazgo llamativo teniendo en cuenta que estudios previos han postulado la hiposmia como un signo preclínico de la enfermedad de Parkinson ${ }^{20}$. Se plantea que estos síntomas referidos en el paciente mayor, sean secundarios a otras alteraciones, y no debidos propiamente a la enfermedad de Parkinson.

Otros síntomas no motores reportados, como los trastornos sensitivos (18.3\%), la depresión (14.3\%) y el dolor $(7.2 \%)$, tienden a ser más frecuentes en pacientes con más de 1 año de evolución de la enfermedad y en aquellos mayores de 50 años. Al comparar con series internacionales, se encuentra una notable diferencia, en las cuales se ha descrito un compromiso de hasta $22.5 \%$ de depresión y $60.9 \%$ de dolor, síntomas que aumentan en frecuencia directamente proporcional a la severidad de la enfermedad ${ }^{14}$.
La demencia, ha sido ampliamente descrita como un síntoma no motor en la enfermedad de Parkinson. Se estima una prevalencia del 20 al $44 \% 21,22$, principalmente en sujetos de edad avanzada ${ }^{23}$ y se cree que tiene una aparición tardía, varios años después de iniciados los síntomas motores 24 . En nuestro estudio $2 \%$ del total de pacientes cursó con cambios demenciales. En $66.6 \%$ de estos casos, los pacientes tenían más de 5 años de evolución de los síntomas motores. Probablemente la baja frecuencia de estos casos se debe a que los datos fueron obtenidos de registros de una primera consulta, y no de un seguimiento prospectivo de estos pacientes.

La principal limitación de nuestro estudio es el tiempo de evolución de la enfermedad al momento de la valoración por el neurólogo experto en trastornos del movimiento, lo cual facilita el sesgo de memoria al momento de recolectar los datos. A futuro se deben incluir otras variables que hacen parte de los síntomas no motores como las disautonomías, la sialorrea y el estreñimiento. Además la severidad de la enfermedad medida por escala de Hoehn y Yahr para correlacionar con los síntomas no motores.

\section{CONCLUSIÓN}

La enfermedad de Parkinson es la segunda enfermedad neurodegenerativa más frecuente a nivel mundial, teniendo una presentación clínica heterogénea. En la población estudiada se encontraron frecuencias de patrones motores similares con los registros internacionales, a excepción del sexo y las manifestaciones no motoras. Es necesaria la realización de un registro nacional de pacientes con la enfermedad de Parkinson para tener una aproximación más exacta de las características de esta enfermedad en la población colombiana.

\section{Conflicto de intereses}

Los autores manifiestan no tener conflictos de intereses en este estudio.

\section{REFERENCIAS}

1. Cohen RM. Epidemiology and Clinical Diagnosis: Alzheimer Disease. PET Clin [Internet]. 2013 Oct [cited 2015 Jul 11];8(4):391405. Available from: http://www.sciencedirect.com/science/ article/pii/S1556859813000631

2. Chen S-Y, Tsai S-T. The Epidemiology of Parkinson's Disease. Tzu Chi Med J [Internet]. 2010 Jun [cited 2015 Jul 11];22(2):73-81. Available from: http://www.sciencedirect.com/science/article/ pii/S1016319010600444

3. De Lau LML, Breteler MMB. Epidemiology of Parkinson's disease. Lancet Neurol [Internet]. 2006 Jun [cited 2014 Oct
3];5(6):525-35. Available from: http://www.sciencedirect.com/ science/article/pii/S1474442206704719

4. Van Den Eeden SK. Incidence of Parkinson's Disease: Variation by Age, Gender, and Race/Ethnicity. Am J Epidemiol [nternet]. 2003 Jun 1 [cited 2015 Apr 3];157(>11):1015-22. Available from: http://aje.oxfordjournals.org/cgi/content/long/157/11/1015

5. Pradilla A. G, Vesga A. BE, León-Sarmiento FE. Estudio neuroepidemiológico nacional (EPINEURO) colombiano. Rev Panam Salud Pública [Internet]. Organización Panamericana de la Salud; 2003 Aug [cited 2015 Jul 11];14(2):104-11. Available from: http:// 
www.scielosp.org/scielo.php?script=sci_arttext\&pid=S102049892003000700005\&lng=en\&nrm=iso\&tlng=es

6. Sánchez JL, Buriticá O, Pineda D, Uribe CS, Palacio LG. Prevalence of Parkinson's disease and parkinsonism in a Colombian population using the capture-recapture method. Int J Neurosci [nternet]. Informa UK Ltd UK; 2004 Feb 7 [cited 2015 Jul 11];114(2):175-82. Available from: http://informahealthcare. com/doi/abs/10.1080/00207450490269444\#.VaGJhd6Zz9o. mendeley

7. Burbulla LF, Krüger R. Converging environmental and genetic pathways in the pathogenesis of Parkinson's disease. J Neurol Sci. 2011;306(1-2):1-8.

8. Johnson KE. Approach to the Patient with Parkinson Disease. Prim Care Clin Off Pract [Internet]. Elsevier; 2015 Jul 11;42(2):205-15. Available from: http://dx.doi.org/10.1016/j. pop.2015.01.005

9. Hughes AJ, Daniel SE, Kilford L, Lees AJ. Accuracy of clinical diagnosis of idiopathic Parkinson's disease: a clinicopathological study of 100 cases. J Neurol Neurosurg Psychiatry. 1992;55(3):181-4.

10. Sung VW, Nicholas AP. Nonmotor symptoms in Parkinson's disease: expanding the view of Parkinson's disease beyond a pure motor, pure dopaminergic problem. Neurol Clin [Internet]. 2013 Aug [cited 2015 Jun 13];31(3 Suppl):S1-16. Available from: http://www.sciencedirect.com/science/article/pii/ S0733861913000522

11. Armstrong MJ, Gruber-Baldini AL, Reich SG, Fishman PS, Lachner C, Shulman LM. Which features of Parkinson's disease predict earlier exit from the workforce? Parkinsonism Relat Disord [Internet]. Elsevier; 2015 Jul 11;20(11):1257-9. Available from: http://dx.doi.org/10.1016/j.parkreldis.2014.08.005

12. Jankovic J., McDermott M., Carter J., et al: Variable expression of Parkinson's disease: A base-line analysis of the DATATOP cohort. Neurology. 1990; 40(10):1529-34.

13. Cervantes-Arriaga A, Rodríguez-Violante M, López-Ruiz M, Estrada-Bellmann I, Zuñiga-Ramírez C, Otero-Cerdeira E, et al. Profile characterization of Parkinson's disease in Mexico: ReMePARK study. Gac Med Mex. 2013;149(5):497-501.

14. Barone P, Antonini A, Colosimo C, Marconi R, Morgante L,
Avarello TP, et al; PRIAMO study group. The Priamo Study: A Multicenter Assessment of Nonmotor Symptoms and Their Impact on Quality of Life in Parkinson's Disease. Mov Disord. 2009;24(11):1641-9.

15. Foltynie T, Brayne C, Barker RA. The heterogeneity of idiopathic Parkinson's disease. J Neurol. 2002;249(2):138-45.

16. van Rooden SM, Colas F, Martínez-Martín P, Visser M, Verbaan D, Marinus J, et al. Clinical subtypes of Parkinson's disease. Mov Disord. 2011;26(1):51-8.

17. Diazgranados JA, Chan L, Gomez LF, Lozano AF, Ramirez M. Descripción de la población de pacientes con enfermedad de Parkinson en un centro médico neurológico en la ciudad de Cali, Colombia. Acta Neurol Colomb. 2011;27(4):205-10.

18. Martínez-Martín P, Hernández B, Ricart J; FAST Study Group. Factors determining when to start levodopa/carbidopa/entacapone treatment in Spanish patients with Parkinson's disease. Neurología. 2014;29(3):153-60.

19. Cervantes-Arriaga A, Rodríguez-Violante M, Camacho-Ordóñez A, González-Latapi P, López-Ruiz M, Estrada-Bellmann I, et al. Time from motor symptoms onset to diagnosis of Parkinson's disease in Mexico. Gac Med Mex. 2014;150(2):242-7.

20. Ponsen MM, Stoffers D, Booij J, van Eck-Smit BL, Wolters ECh, Berendse HW. Idiopathic hyposmia as a preclinical sign of Parkinson's disease. Ann Neurol. 2004;56(2):173-81.

21. Martinez-Martin P, Schapira AH, Stocchi F, et al. Prevalence of nonmotor symptoms in Parkinson's disease in an international setting; study using nonmotor symptoms questionnaire in 545 patients. Mov Disord 2007;22(11):1623-9.

22. Truong DD1, Bhidayasiri R, Wolters E. Management of nonmotor symptoms in advanced Parkinson disease. J Neurol Sci. 2008;266(1-2):216-28.

23. Hughes TA, Ross HF, Musa S, Bhattacherjee S, Nathan RN, Mindham RH, et al. A 10-year study of the incidence of and factors predicting dementia in Parkinson's disease. Neurology. 2000;54(8):1596-602.

24. Sung VW, Nicholas AP. Nonmotor symptoms in Parkinson's disease: expanding the view of Parkinson's disease beyond a pure motor, pure dopaminergic problem. Neurol Clin. 2013;31(3 Suppl):S1-16. 